\section{THE DETERMINATION OF METEOR AND ATMOSPHERIC PARAMETERS USING RADAR AND DIGITAL TECHNIQUES}

\author{
Lynden J Rogers \\ Department of Physics, University of Newcastle, Newcastle \\ NSW 2308 \\ July 1991
}

The continued development of a micro-processor based data logger for use in conjunction with the radar meteor system at Newcastle is discussed. The essential algorithms required for noise rejection, meteor profile recognition, and the extraction of meteor and atmospheric parameters are presented. Essential system parameters are detailed, as are the procedures employed for calibrating and monitoring system performance.

Data from two operating periods, 1987 and 1990 respectively, are presented and discussed. This data is related to established theory, and compared with relevant published results from other studies where appropriate.

Although high noise conditions greatly hampered the observations, the digital system was demonstrated to function reliably and the algorithms were shown to produce useful and consistent data. A system comprised of dedicated microprocessors, reducing data in real time, is suggested as one of the best, while certainly the most cost effective real time meteor loggers available using current technology.

\section{LINEAR ACCELERATION EMISSION}

\section{Eric Thomas Rowe}

Department of Theoretical Physics University of Sydney NSW 2005

\section{September 1992}

The aim of this thesis is to explore in detail linear acceleration emission by charged particles moving in the field of a longitudinal plasma wave. The motivation of the work is primarily the possible application of this emission mechanism to explaining part or all of the radio emission from pulsars. Due to the close connection of this work to the theory of double layers, there is also the possibility of application to other areas of astrophysics. Here we consider only the application to pulsars.

In Chapter 1 we treat both trapped and untrapped particle motion in a sinusoidal wave field with subluminal phase speed $\left(v_{\phi}<c\right)$. We include both the effect of the wave field and relativistic effects exactly. In Chapter 2 the results are extended to superluminal $\left(v_{\phi}>c\right)$ and luminal $\left(v_{0}=c\right)$ waves. The Fourier transformed single particle current density is calculated in Chapter 3 for each type of particle motion and expressions for the power emitted by the particles are given. In particular the power spectrum for untrapped particles in subluminal waves and for particles in superluminal waves are discussed and compared.

The theory of large amplitude waves in an arbitrary plasma is summarised in general for subluminal and superluminal waves in Chapter 4. Exact results for longitudinal waves in an electron-positron plasma with 'flat' distributions in momentum, enable comparison between the subluminal and superluminal cases. Particle orbits in large amplitude waves may be characterised by the drift motion of the particle which describes the motion of the centre of oscillation of the particle (the centre of oscillation is analogous to the centre of gyration of a particle in a magnetic field). The distribution of particles in this drift momentum may be used to derive the emission and absorption coefficients for radiation emitted by the particles. This distribution is derived in the superluminal wave case for a large amplitude wave with an almost sinusoidal profile.

In Chapter 5 the quasilinear theory for the calculation of emission and absorption coefficients in an arbitrary plasma is discussed. The theory is then applied to a plasma in which there is a large amplitude wave. The superluminal wave case is discussed in detail. In Chapter 6 the averaged emission and absorption coefficients are given for the superluminal wave case. In particular we develop the theory for weak wave-particle interaction and for strong wave-particle interaction.

Chapter 7 deals with the application of linear acceleration emission to radio pulsars. After a brief overview of pulsars, including their discovery and identification with rotating neutron stars, the characteristics of their emission and aspects of pulsar magnetospheres, the growth rate possible for the linear acceleration mechanism is calculated in the context of pulsars. From the high brightness temperatures of pulsar radio emission it is inferred that a coherent emission process is responsible for the emission. A coherent mechanism requires that the optical depth $\tau \ll-1$. In the case of strong wave-particle interaction, it is found that the growth rate is sufficient to imply coherent emission over the entire range of pulsar emission. In particular, for one choice of parameters $\tau$ varies between $\approx 2 \times 10^{4}$ and $\approx-10^{3}$ over the range of pulsar frequencies, while for another choice of parameters $\tau$ varies between $\approx-200$ and $\approx-10$.

\section{STUDIES OF THE FAINT GALAXY POPULATION}

\section{Boudewijn F. Roukema}

Mount Stromlo and Siding Spring Observatories, Australian

National University, Canberra ACT 2601

January 1993

Recent observations of faint galaxies to $b_{\mathbf{J}} \approx 28$ (e.g., Tyson $\&$ Seitzer, 1988) show an excess in number density with respect to simple flat universe models which incorporate $\mathrm{K}$ and E-corrections but unevolving luminosity functions. Low $q_{0}$, high $z_{f}$ models are unfavoured by recent redshift measurements, but merging dominated models and models involving differential evolution between bright and faint galaxies or a new population of faint galaxies remain consistent with the data and flat universe.

In this thesis, observations and theory which contribute to our understanding of these faint galaxy populations are described. In chapter 2 it is shown that $d_{L}, d V / d z, q_{0}, z_{f}$, the $\mathrm{K}$ - and E-corrections, $\emptyset^{*}, M^{*}, a$, and $\eta$ all affect the faint number counts significantly, though not independently, while the effect of $H_{\mathrm{o}}$ is small.

A preliminary search for low surface brightness galaxies described in Chapter 3 gave unpromising results, with a number density to $z \approx 0.05$ of $\eta \approx(9 \pm 5) \times 10^{-3} h^{3} \mathrm{Mpc}^{-3}$, which is about $7 \pm 4 \%$ of the number density for normal galaxies in the corresponding magnitude range of $-14 \geq M_{B}$ $\geq-20$ represented in a Schechter (1976) luminosity function with Efstathiou et al.'s (1988) parametrisation. Only about half of this low surface brightness galaxy population is likely to be excess to that represented in the Schechter function. The diameters of the population observed are inconsistent with the hypothesis that they are the low-redshift 